\title{
Model-based Fault Diagnostics of Servo Valves
}

\author{
Turki Haj Mohamad ${ }^{1}$, Foad Nazari² ${ }^{2}$ and Chandrasekhar Nataraj ${ }^{3}$ \\ 1,2,3 Villanova Center for Analytics of Dynamic Systems (vcads.org), \\ Villanova University, Villanova, PA 19085, USA \\ thajmoha@villanova.edu \\ foad.nazari@villanova.edu \\ c.nataraj@villanova.edu
}

\begin{abstract}
This paper presents the application of the Extended Phase Space Topology (EPST) method in model-based diagnostics of nonlinear systems. A detailed nonlinear mathematical model of a servo electro-hydraulic system has been used to demonstrate the procedure. Two faults have been considered associated with the servo valve including the increased friction between spool and sleeve and the degradation of the permanent magnet of the valve armature. The faults have been simulated in the system by the variation of the corresponding parameters in the model and the effect of these faults on the output flow response has been investigated. A regression-based artificial neural network has been developed and trained using the EPST extracted features to estimate the original values of the faulty parameters and to identify the severity of the faults in the system.
\end{abstract}

\section{INTRODUCTION}

Electro-hydraulic servo valves have generated considerable research interest due to their high degree of accuracy in regulating and controlling fluid flow in a wide variety of applications such as hydro-electric power plants, aircraft engines and manufacturing. Servo valves consist of complex components that handle precise and sensitive tasks, such as starting or stopping a flow, modifying the velocity or changing the pressure, etc. Any deviation of the system components' parameters or dimensions may lead to instability or system failure. Hence, it is important to develop effective diagnostic techniques to constantly monitor the performance of such systems and to identify any faults, including their locations and severity levels.

Fault detection and diagnostic techniques can be classified into two main categories: data driven approaches and modelbased approaches. Data driven approaches are based on sig-

\footnotetext{
Turki Haj Mohamad et al. This is an open-access article distributed under the terms of the Creative Commons Attribution 3.0 United States License, which permits unrestricted use, distribution, and reproduction in any medium, provided the original author and source are credited.
}

nal processing techniques that are performed on data measured from the system. These techniques strive to extract features to indicate the status of the system. Model-based approaches use physics, represented by mathematical models of the system, in order to detect faults.

One of the main approaches in model-based fault detection is to use parameter estimation techniques (Isermann, 1982, 1984; Frank, Ding, \& Koppen-Seliger, 2000). Parameter estimation is the main scope of this research, which is based on estimating and comparing the parameters of a defective system with the parameters of a healthy system. The main thesis is that the change in the system parameters is usually associated with the system defects (Isermann, 2005; Baskiotis, Raymond, \& Rault, 1979; Kappaganthu \& Nataraj, 2011; Liu, Zhang, Liu, \& Yang, 2000).

Due to the highly nonlinear characteristics of servo valves, it is essential to use techniques that can perform effectively in different domains of the nonlinear response. This paper presents a continuation of our past work (Samadani, Kwuimy, \& Nataraj, 2014), in which the application of recurrence plots (RPs) and recurrence quantification analysis (RQA) were introduced. Despite the success of the previous method in parameter estimation, it can be time consuming and computationally demanding, which makes it hard for it to be applied in an automated manner or for it to be integrated into a control system. Thus, this investigation presents a new approach for parameter estimation-based diagnostics of nonlinear systems, based on the extracted information from the nonlinear response.

In an earlier work, we presented the method of Phase Space Topology (PST) (Samadani, Kwuimy, \& Nataraj, 2015, 2013), which is based on transforming the phase space into the density space and characterizing the density with quantitative measures. It was shown that, depending on the geometry and shape of the phase space, the density profile contains peaks of various heights and sharpness at multiple locations. The properties of the peaks in the density distri- 
bution, including the location, height and sharpness of the peaks, were used as features in the initial approach. The need to search for the peaks in the density distributions makes the PST difficult or sometimes even impractical to implement, especially for systems with noisy or more complex phase space patterns. We next improved this approach with Extended Phase Space Topology (EPST) (Mohamad \& Nataraj, 2017; Samadani, Mohamad, \& Nataraj, 2016; Mohamad, Samadani, \& Nataraj, n.d.; Mohamad, Kwuimy, \& Nataraj, 2017), which will be discussed in detail in Section 3. The EPST method is based on characterizing the topology of the density distribution of the response signal, which is then expanded in a series of Legendre polynomials. The coefficients of the orthogonal polynomials are subsequently used as features for a machine learning algorithm to estimate the system parameters. The present paper extends that approach to be used as a parameter estimation model-based technique.

Model-based condition monitoring requires a mathematical representation of the system that can describe the behavior of the system (here, the servo valve). Thus, a detailed nonlinear mathematical model has been used to simulate the system response under various conditions. Faults can be defined as undesired deviations of a characteristic property or a system parameter from the standard acceptable domain, which causes limitations in achieving a planned task (Isermann, 1984). Two faults have been considered associated with the servo valve: the increased friction between spool and sleeve and the degradation of the permanent magnet of the valve armature. Various electrical current signals, i.e., periodic, biperiodic and quasi-periodic, are used as inputs to the servo valve system. The EPST method is then performed on the output flow of the servo valve to identify dynamical changes in the system associated with the faults. Finally, an artificial neural network (ANN) is trained for mapping of the feature space to the parameter space.

The remaining parts of this paper are organized as follows. In Section 2, a detailed mathematical model of the electrohydraulic valve has been derived. In Section 3, the definition of EPST has been presented. Section 4 presents a parametric analysis for the servo valve system. In Section 5, the parametric estimation algorithm is discussed along with the results. Finally, Section 6 concludes the paper.

\section{Modeling of The Electro-Hydraulic Servo SYSTEM}

A detailed dynamical model of a two-stage servo valve system (shown in Fig. 1) with mechanical feedback has been used in the analysis. Only the final equations are presented here. The detailed explanation of the model derivations can be found in (Samadani, Behbahani, \& Nataraj, 2013; Rabie, 2009; Gordić, Babić, \& Jovičić, 2004). The definition of sys-

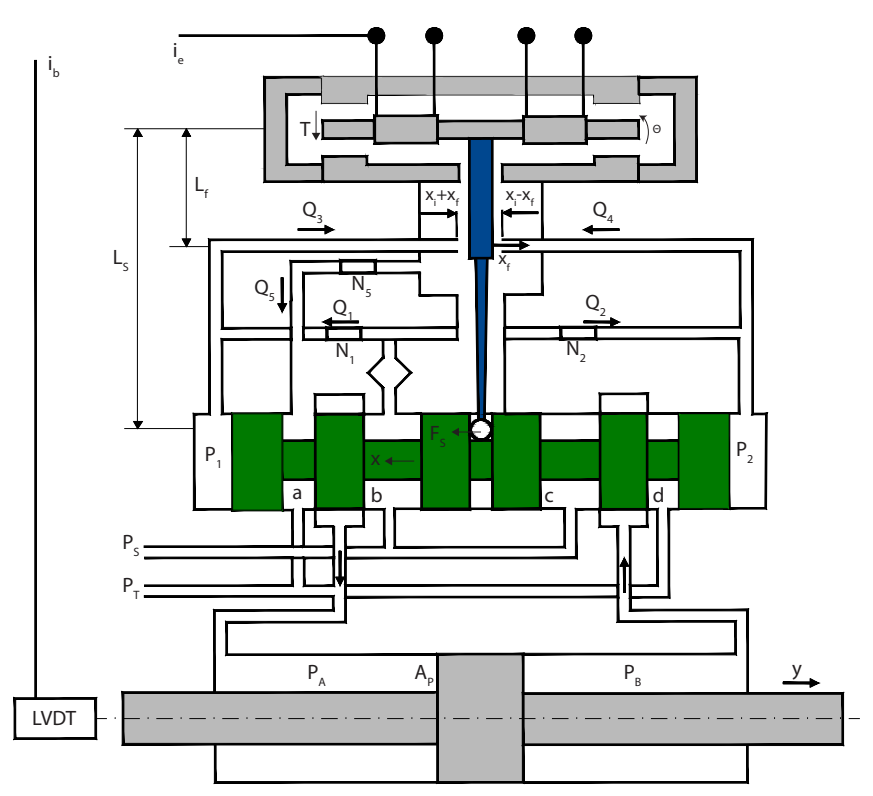

Figure 1. Functional schematic of the electro-hydraulic servo system

tem states and parameters along with nominal values of the parameters have been presented in the nomenclature.

Neglecting the effect of the magnetic hysteresis, the net torque on the armature is given by the following expression.

$$
T=K_{i} i_{e}
$$

where, the coefficient $K_{i}$ can be calculated by:

$$
K_{i}=\frac{N \lambda_{p} \mu_{o} A L}{2 x_{o}^{2}}
$$

The motion of the armature and the elements attached to it is described by the following equations:

$$
\begin{gathered}
T=J \frac{d^{2} \theta}{d t^{2}}+f_{\theta} \frac{d \theta}{d t}+K_{T} \theta+T_{L}+T_{P}+T_{F} \\
T_{P}=A_{f}\left(P_{2}-P_{1}\right) L_{f}
\end{gathered}
$$

where,

$$
A_{f}=\frac{\pi}{4} d_{f}^{2}
$$

The feedback torque depends on the displacement of the spool and the angle of the flapper and can be given by:

$$
T_{F}=F_{S} L_{S}=K_{S}\left(L_{S} \theta+x\right) L_{S}
$$

The rotational displacement of the flapper is limited mechanically by the jet nozzles. When the flapper reaches any of the side jet nozzles, a counter torque $T_{L}$ is applied to it which can 
be calculated by the following equation:

$$
T_{L}= \begin{cases}0, & \left|x_{f}\right|<x_{i} \\ R_{s} \frac{d \theta}{d t}-\left(\left|x_{f}\right|-x_{i}\right) K_{L f} L_{f} \operatorname{sign}\left(x_{f}\right), & \left|x_{f}\right|>x_{i}\end{cases}
$$

where,

$$
x_{f}=L_{f} \theta
$$

The flow rates through the flapper valve restrictions are given by the following equations:

$$
\begin{aligned}
Q_{1} & =C_{D} A_{o} \sqrt{\frac{2}{\rho}\left(P_{s}-P_{1}\right)}=C_{12} \sqrt{\left(P_{s}-P_{1}\right)} \\
Q_{2} & =C_{D} A_{o} \sqrt{\frac{2}{\rho}\left(P_{s}-P_{2}\right)}=C_{12} \sqrt{\left(P_{s}-P_{2}\right)} \\
Q_{3} & =C_{d} \pi d_{f}\left(x_{i}+x_{f}\right) \sqrt{\frac{2}{\rho}\left(P_{1}-P_{3}\right)} \\
& =C_{34}\left(x_{i}+x_{f}\right) \sqrt{\left(P_{1}-P_{3}\right)} \\
Q_{4} & =C_{d} \pi d_{f}\left(x_{i}-x_{f}\right) \sqrt{\frac{2}{\rho}\left(P_{2}-P_{3}\right)} \\
& =C_{34}\left(x_{i}-x_{f}\right) \sqrt{\left(P_{2}-P_{3}\right)} \\
Q_{5} & =C_{d} A_{s} \sqrt{\frac{2}{\rho}\left(P_{3}-P_{T}\right)}=C_{5} \sqrt{\left(P_{3}-P_{T}\right)}
\end{aligned}
$$

By using the continuity equation for the chambers of the flapper valve, the following expressions can be deduced:

$$
\begin{aligned}
Q_{1}-Q_{3}+A_{s} \frac{d x}{d t} & =\frac{V_{o}-A_{s} x}{B} \frac{d P_{1}}{d t} \\
Q_{2}-Q_{4}-A_{s} \frac{d x}{d t} & =\frac{V_{o}+A_{s} x}{B} \frac{d P_{2}}{d t} \\
Q_{3}+Q_{4}-Q_{5} & =\frac{V_{3}}{B} \frac{d P_{3}}{d t}
\end{aligned}
$$

The motion of the spool is governed by the following equations.

$$
\begin{aligned}
& A_{s}\left(P_{2}-P_{1}\right)=m_{s} \frac{d^{2} x}{d t^{2}}+f_{s} \frac{d x}{d t}+F_{j}+F_{s} \\
& F_{j}= \begin{cases}\left(\frac{\rho Q_{b}^{2}}{C_{c} A_{b}}+\frac{\rho Q_{d}^{2}}{C_{c} A_{d}}\right) \operatorname{sign}(x) & \text { for } x>0 \\
\left(\frac{\rho Q_{a}^{2}}{C_{c} A_{a}}+\frac{\rho Q_{c}^{2}}{C_{c} A_{c}}\right) \operatorname{sign}(x) & \text { for } x<0\end{cases}
\end{aligned}
$$

Ignoring the effect of transmission lines between the valve and the symmetrical hydraulic cylinder, the flow rates through the valve restriction areas are given by:

$$
\begin{aligned}
Q_{a} & =C_{d} A_{a}(x) \sqrt{\frac{2}{\rho}\left(P_{A}-P_{T}\right)} \\
Q_{b} & =C_{d} A_{b}(x) \sqrt{\frac{2}{\rho}\left(P_{s}-P_{A}\right)} \\
Q_{c} & =C_{d} A_{c}(x) \sqrt{\frac{2}{\rho}\left(P_{s}-P_{B}\right)} \\
Q_{d} & =C_{d} A_{d}(x) \sqrt{\frac{2}{\rho}\left(P_{B}-P_{T}\right)}
\end{aligned}
$$

The area of the valve restrictions are given by:

$$
\begin{aligned}
& \begin{cases}A_{a}=A_{c}=\omega c & \text { for } x \geq 0 \\
A_{b}=A_{d}=\omega \sqrt{\left(x^{2}+c^{2}\right)} & \end{cases} \\
& \begin{cases}A_{a}=A_{c}=\omega \sqrt{\left(x^{2}+c^{2}\right)} & \text { for } x \leq 0 \\
A_{b}=A_{d}=\omega c & \end{cases}
\end{aligned}
$$

Considering the internal leakage and neglecting the external leakage, the following equations can be obtained by applying the continuity equation to the cylinder chambers.

$$
\begin{aligned}
& Q_{b}-Q_{a}-A_{P} \frac{d y}{d t}-\frac{\left(P_{A}-P_{B}\right)}{R_{i}}=\frac{\left(V_{c}+A_{p} y\right)}{B} \frac{d P_{A}}{d t} \\
& Q_{c}-Q_{a} d+A_{P} \frac{d y}{d t}-\frac{\left(P_{A}-P_{B}\right)}{R_{i}}=\frac{\left(V_{c}-A_{p} y\right)}{B} \frac{d P_{B}}{d t}
\end{aligned}
$$

Finally, the equation of motion for the cylinder piston is given by:

$$
A_{P}\left(P_{A}-P_{B}\right)=m_{p} \frac{d^{2} y}{d t^{2}}+f_{P} \frac{d y}{d t}+K_{b} y
$$

\section{Feature Extraction Method}

Let $X=\left(x_{1}, x_{2}, \ldots, x_{n}\right)$ be an independent and identically distributed sample data drawn from a distribution with an unknown density function $\Psi$. The shape of this function can be estimated by its kernel density estimator (indicates that it is an estimate, and $h$ indicates that its value can depend on $h$ ).

$$
\hat{\Psi}_{h}(x)=\frac{1}{n h} \sum_{i=1}^{n} \Gamma\left(\frac{x-x_{i}}{h}\right)
$$

where, $h>0$ is a smoothing parameter called the bandwidth, and $\Gamma($.$) is the kernel function which satisfies the following$ requirements.

$$
\int_{-\infty}^{\infty} \Gamma(u) d u=1
$$




$$
\Gamma(-u)=\Gamma(u) \forall u
$$

There is a range of kernel functions that can be used, including uniform, triangular, biweight, triweight, Epanechnikov, normal, etc. Due to its conventional and convenient mathematical properties, we use the standard normal density function in our approach, defined as the following:

$$
\Gamma(u)=\frac{1}{\sqrt{2 \pi}} e^{-\frac{1}{2} u^{2}}
$$

Let $x$ be a state of the system and $y_{d}=\hat{f}_{h}(x)$, its density computed using the kernel density estimator. $y_{d}$ is then approximated with Legendre orthogonal polynomials. Legendre polynomials can be directly obtained from Rodrigues' formula which is given by:

$$
\Phi_{m}(x)=\frac{1}{2^{m} m !} \frac{d^{m}}{d x^{m}}\left[\left(x^{2}-1\right)^{m}\right], \quad m=0,1,2, \ldots
$$

It can also be obtained using Bonnet's recursion formula:

$$
(m+1) \Phi_{m+1}(x)=(2 m+1) x \Phi_{m}(x)-m \Phi_{m-1}(x)
$$

where the first two terms are given by:

$$
\Phi_{0}(x)=1, \quad \Phi_{1}(x)=x
$$

The coefficients of the Legendre polynomials are obtained by using the least squares method assuming the following linear regression model:

$$
\Psi(x, \beta)=\sum_{j=1}^{m} \beta_{j} \Phi_{j}(x)
$$

Letting

$$
X_{i j}=\frac{\partial \Psi\left(x_{i}, \boldsymbol{\beta}\right)}{\partial \beta_{j}}=\Phi_{j}\left(x_{i}\right)
$$

the estimated coefficients are given by:

$$
\hat{\beta}=\left(X^{T} X\right)^{-1} X^{T} y_{d}
$$

The coefficients $\hat{\beta}$ constitute the features in our approach that can be used in classification or regression problems. The approximated density using Legendre Polynomials is then calculated using the following:

$$
\Psi_{a}=X \hat{\beta}
$$

Root mean square error (RMSE) and Pearson's correlation coefficient (PCC) were calculated to compute the quality of the fit using the following equations:

$$
\mathrm{RMSE}=\sqrt{\frac{1}{N} Z Z^{T}}, \quad \mathrm{PCC}=\frac{\sigma_{d}^{T} \sigma_{a}}{\sqrt{\left(\sigma_{d}^{T} \sigma_{d}\right)\left(\sigma_{a}^{T} \sigma_{a}\right)}}
$$

where, $Z=\left(y_{d}-\Psi_{a}\right)$ is the residual vector, $N$ is the number of points in the density function, $\sigma_{d}=\left(y_{d}-E\left\{y_{d}\right\}\right)$ and $\sigma_{a}=\left(\Psi_{a}-E\left\{\Psi_{a}\right\}\right) . E\{$.$\} is the expected value.$

\section{Parametric analysis}

For identification of faults, we propose to inject a prespecified signal to the system and to compare the system response with the healthy condition response. In this study, an electrical current signal is applied to the servo valve system after which the output flow of the servo valve is measured for one second. Note that all analysis were performed using the mathematical model without any experimental data.

To analyze the effectiveness of the approach, three signals were used as inputs for the servo valve as follows:

- Periodic input signal

$$
i=0.01 \sin (50 t)
$$

- Bi-Periodic input signal

$$
i=0.01 \sin (50 t)+0.005 \sin (75 t)
$$

- Quasi-Periodic input signal

$$
i=0.01 \sin (50 t)+0.005 \sin (50 \pi t)
$$

There are many parameters that can be monitored in typical electro-hydraulic systems. In this study, as mentioned earlier, two faults have been considered: (1) the degradation of the permanent magnet of the valve armature, represented by the change of the coefficient $K_{i}$, and (2) the increased friction between spool and sleeve, represented by the change of the spool friction coefficient $f_{S}$.

In equations 44 and $45, C K_{i}$ and $C f_{s}$ are defined to show variation of $K_{i}$ and $f_{s}$ from the healthy condition.

$$
\begin{gathered}
C K_{i}=\frac{K_{i}}{\bar{K}_{i}} \\
C f_{s}=\frac{f_{s}}{\bar{f}_{s}}
\end{gathered}
$$

where, $\bar{K}_{i}$ and $\bar{f}_{s}$ denote the values of $K_{i}$ and $f_{s}$ for the healthy condition and are equal to $0.559 \mathrm{Nm} / \mathrm{A}$ and 3.05 Ns/m, respectively. When $C K_{i}$ or $C f_{s}$ are close to 1 , the system can be considered to operate in healthy condition. In addition to the healthy condition $\left(C K_{i}\right.$ and $C f_{s}$ are equal to 1$)$, eight faulty conditions are considered in this section. Four of the conditions are caused by decreasing $C K_{i}$ by $20 \%, 40 \%$, $60 \%$ and $80 \%$. The remaining four conditions are caused by increasing $C f_{s} 5,10,20$ and 30 times.

The effect of the five cases of the coefficient $C K_{i}\left(C K_{i}=\right.$ $\{1,0.8,0.6,0.4,0.2\})$, on the output flow of the servo valve for three input signals are compared in Fig. 2. As is clearly seen in this figure, for all three input cases, a decrease of $C K_{i}$ 
results in a decrease of the output flow amplitude of the servo valve. It is also concluded that the output flow is very sensitive to the variation of $C K_{i}$, because any change in $C K_{i}$ affects the time response of the system.

In Fig. 3, the time response of the servo valve system for three input signals and various values of $C f_{s}$ are presented. Five cases of the $C f_{s}$ parameter were studied $\left(C f_{s}=\right.$ $\{1,5,10,20,30\})$. It can be seen that the effect of $C f_{s}$ on the time response of the system is not as easily observed as the response for changing $C K_{i}$. To show how the output flow of the valve is affected by $C f_{s}$, some main points of the graphs are magnified and added to the main figures. From the magnified sections, it is concluded that increasing the parameter $C f_{s}$ results in a decrease of the amplitude of output flow at certain time ranges and an increase of the output flow amplitude at other ranges. This indicates that changing $C f_{s}$ affects the output flow in a nonlinear fashion. In general, the change in $K_{i}$ or $f_{s}$ affects the output flow of the servo valve system. This indicates that the output flow has valuable information about the parameters $K_{i}$ and $f_{s}$, which can be used in solving the inverse problem of estimating the parameters using all provided information that is contained within the output flow of the servo valve.

\section{FAULT IDENTIFICATION AND SEVERITY ANALYSIS}

The previous section illustrates how the time response of the system is affected by the change of $f_{s}$ and $K_{i}$ parameters and how the response for the $f_{s}$ parameter changes in a nonlinear fashion. In this section, the values of $f_{s}$ and $K_{i}$ parameters are predicted based on the time response of the system. In order to capture all available information provided by the output flow of the servo valve, the EPST method is applied. A summary of the proposed method is shown in Fig. 4.

The EPST extracted features were used to train two artificial neural networks (ANNs). ANNs are a form of a multiprocessor system with a high degree of inter-connection simple processing elements, simple scalar messages and adaptive interaction between elements. A multi-layer feed forward (MLFF) ANNs, which is one of the most popular type of ANNs is used in this study. The structure of the MLFF network includes an input layer, one or more hidden layers and an output layer, in which each layer consists of some neurons.

In this work, the Back-Error Propagation (BEP) algorithm was employed in order to minimize the error of the ANN model. The BEP is one of the most widely used learning algorithm of MLFF-ANNs. In the trained ANNs, the activation functions for the neurons of hidden and output layers were Tansig and linear, respectively. Two distinct ANNs were created with ten neurons in the input layer and a single neuron in the output layer. The inputs of the ANNs were selected to be the first ten coefficients of the Legendre polynomials. The target outputs of first and second ANNs were $C f_{s}$ and

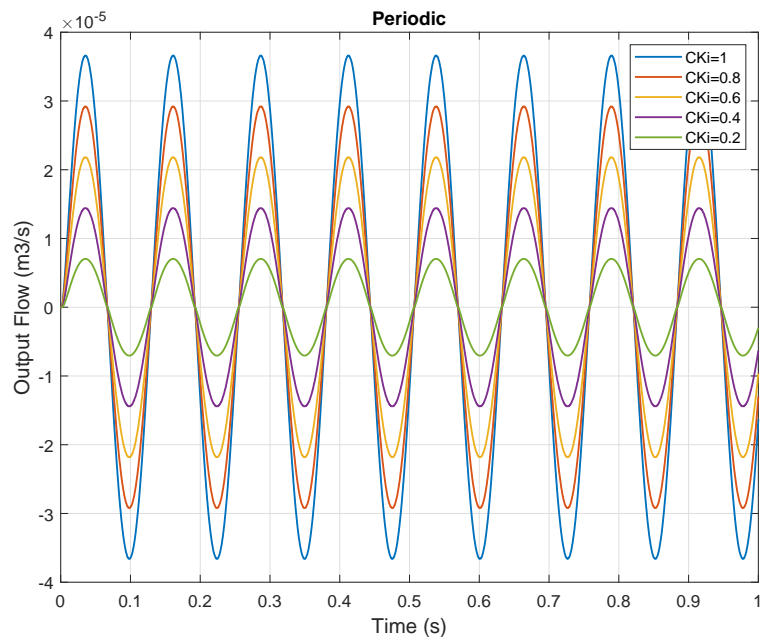

(a)

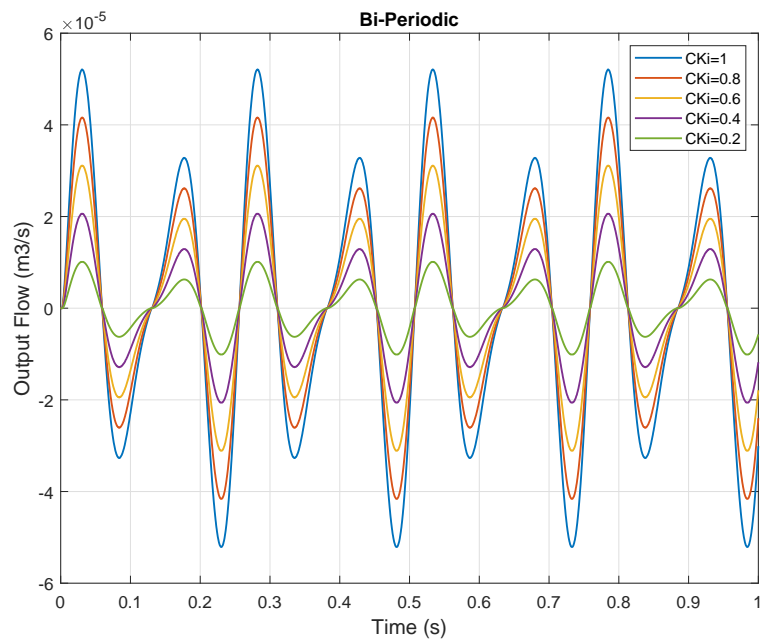

(b)

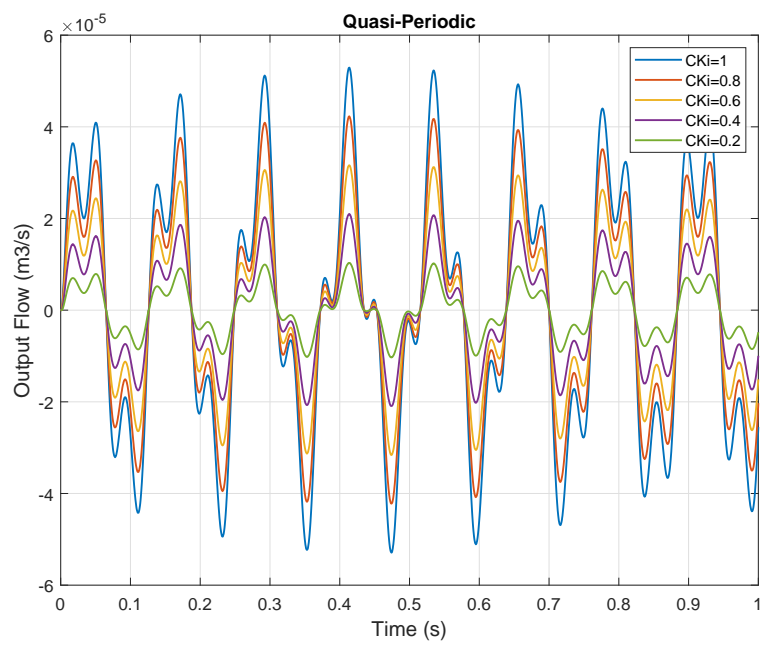

(c)

Figure 2. Time response of the system for (a): periodic, (b): bi-periodic and (c): quasi-periodic inputs to the servo valve for various values of $C K_{i}$ 


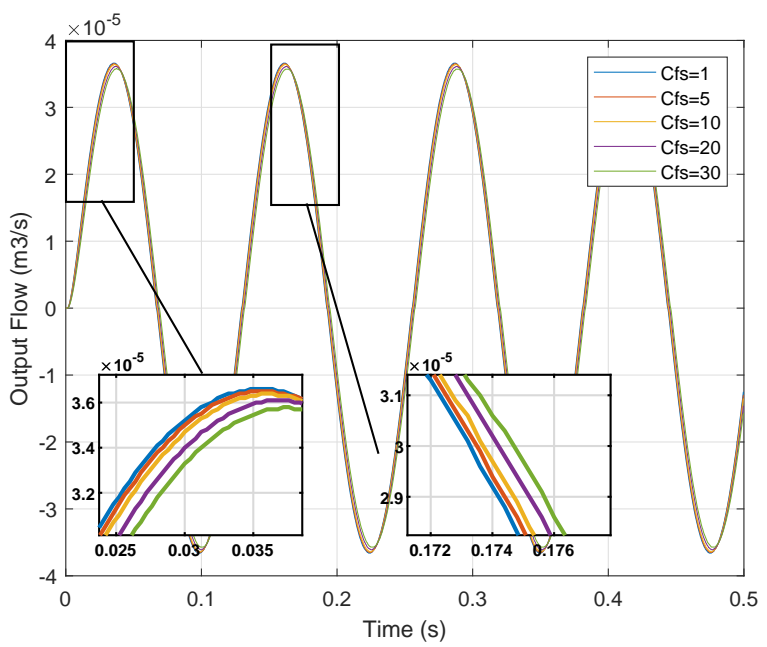

(a)

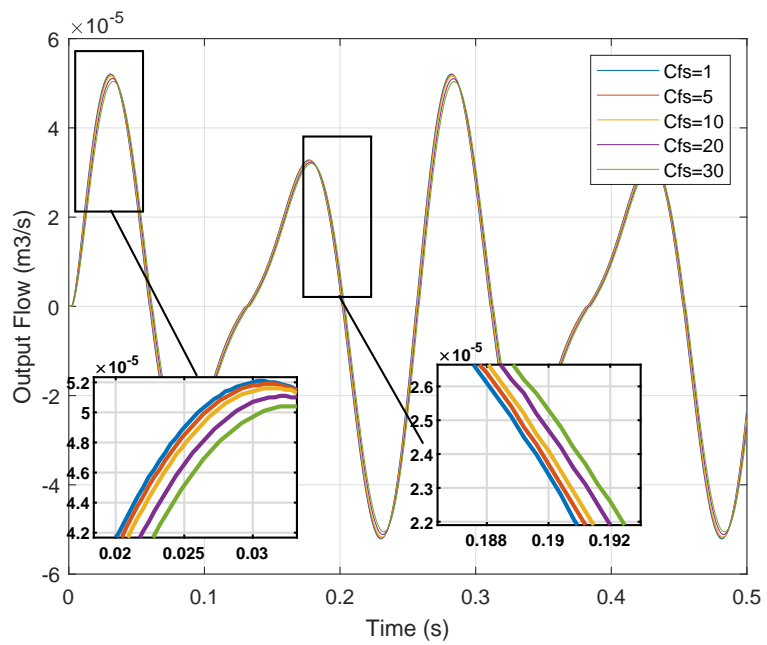

(b)

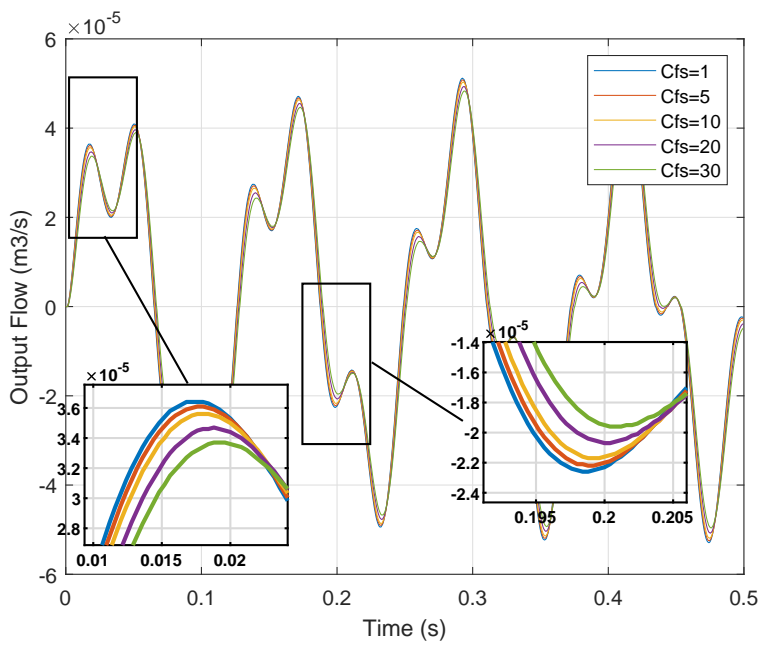

(c)

Figure 3. Time response of the system for (a): periodic, (b): bi-periodic and (c): quasi-periodic inputs to the servo valve for various values of $C f_{s}$

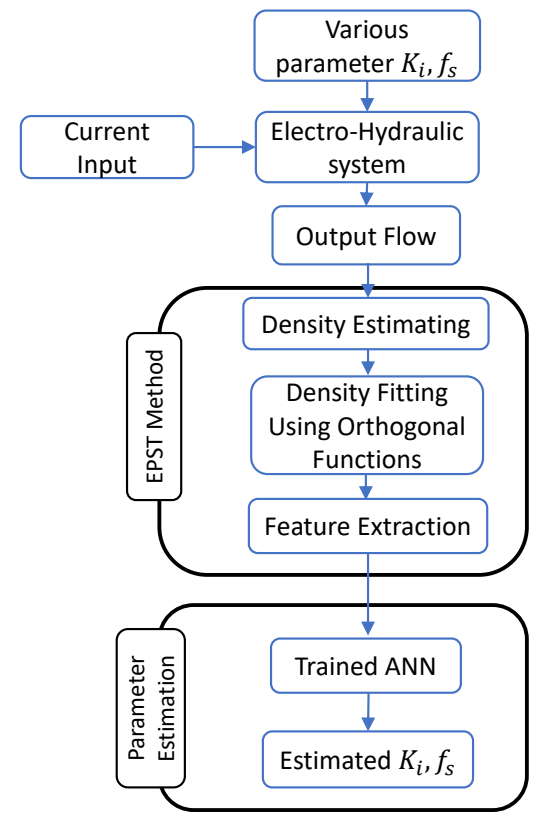

Figure 4. The process of the parameter estimation method

$C K_{i}$, respectively. The data was obtained by random selection of the $f_{s}$ and $K_{i}$ values in the intervals of $[1,100]$ and $[0.1,0.6]$, respectively. Simulation of the servo valve system was then performed and the response features, i.e., Legendre polynomial coefficients, were computed at each time. A total number of 291 samples was used for training, validation and testing of the model. In the BEP training procedure, $70 \%, 20 \%$ and $10 \%$ of data were used for training, testing and verification, respectively. An additional 9 samples were used to compare the predicted and the actual values of the $K_{i}$ and $f_{s}$ parameters. All data were applied to ANNs in normalized form. The ANNs were trained and tested with three various set of data obtained from periodic, bi-periodic and quasi-periodic inputs of the servo-valve system.

To predict the $f_{s}$ and $K_{i}$ parameters, five hidden layer configurations (i.e., 5, 10, 15, 20 or 25 neurons) were considered. Using the obtained data of the quasi-periodic input, a comparison between the different hidden layer configurations was performed. The minimum error was considered to select the optimum neuron number in the hidden layer. The obtained results are presented in Fig. ??. As can be seen, the least error values in prediction of $K_{i}$ and $f_{S}$ were obtained with 15 and 5 neurons in the hidden layer, respectively. The rest of the analyses were performed by considering the obtained optimum neuron numbers for ANNs hidden layer.

The performances of the trained ANN models to predict $K_{i}$ and $f_{s}$ parameters for periodic input are shown in Fig. 5. In the training procedure, the number of epochs was assumed to be 400 . For various input signals the trained ANN was capable of predicting the $K_{i}$ and $f_{s}$ values with high accu- 


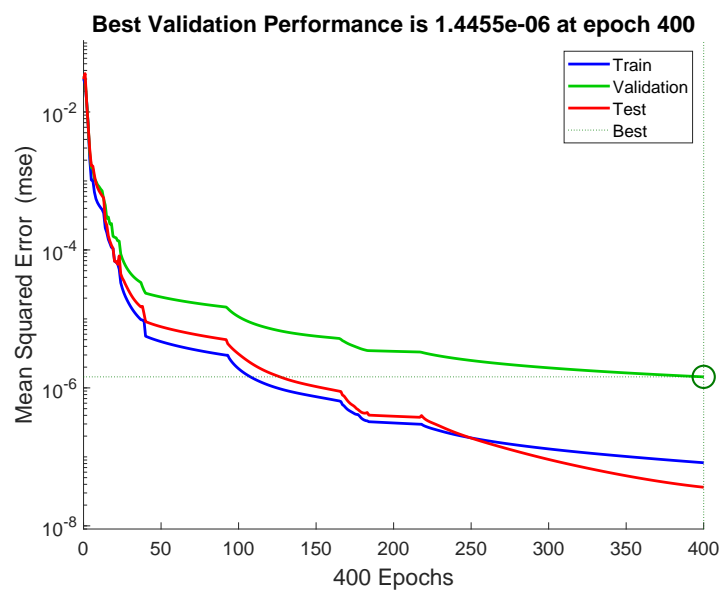

(a)

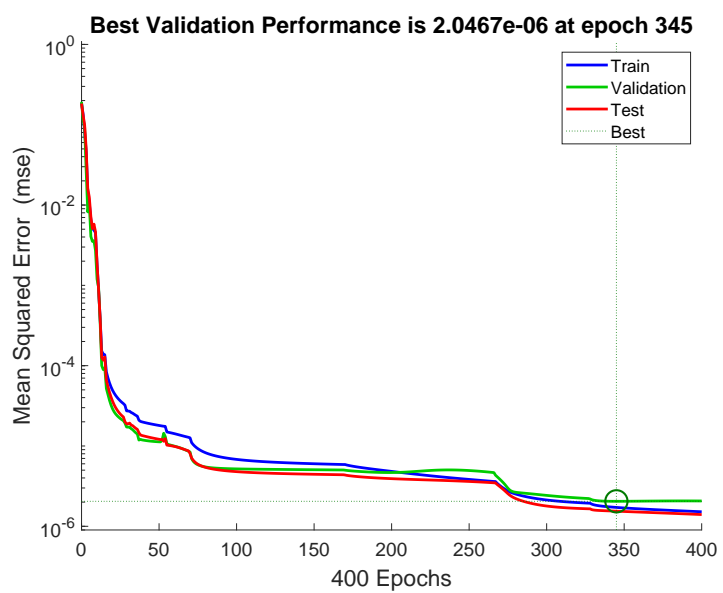

(b)

Figure 5. Training performance of ANNs in prediction of periodic input for a) $K_{i}$ and b) $f_{s}$

racy. This is represented in the performance plots by very low validation and test errors. For example, predicting $K_{i}$ for a periodic input achieved misprediction rate of $1.44 \mathrm{e}-06$ for the validation samples. In addition, Figure. 6 illustrates the regression plots of the network outputs with respect to targets along the regression values for periodic input. Ideally, the regression value should be close to 1 and the data in the regression plot should fall along a $45^{\circ}$ line for a perfect fit. As can be seen in this figure, all the points have fallen along the $45^{\circ}$ line and the regression values are equal or close to 1 , which indicates an accurate mapping of the feature space to the parameter space.

Finally, the predicted results are compared with the actual values in Table 1 . As can be calculated from the prediction results presented in Table 1, the average prediction error for $K_{i}$ and $f_{s}$ are $0.07 \%$ and $0.63 \%$, respectively. This shows that the proposed method has a very good ability to predict the original parameters of the system. Although the parametric analysis showed that the effects of $f_{s}$ on time response of the system was nonlinear and not as easily observed as $K_{i}$, the EPST method was able to predict its value for various input signals and on a wide range with $99.37 \%$ accuracy. The EPST parameter estimation algorithm is virtually perfect in predicting the $K_{i}$ value with $99.93 \%$ accuracy.

\section{Conclusion}

We used the Extended Phase Space Topology (EPST) method for model-based fault detection and diagnostics of an electrohydraulic system. It was shown that the nonlinear response of the system contains valuable information about the system that can be used for this purpose. The analyses were performed with the assumption that only the output response of the system (here output flow of the valve) is available. A parameter analysis was performed on the system in order to study the relationship between changing $K_{i}$ and $f_{s}$ parameters and the system response. It was shown that $K_{i}$ affects the system response linearly where increasing the parameter leads to an increase in the output flow amplitude. On the other hand, changing $f_{s}$ has a nonlinear relationship to the output flow of the system. It was demonstrated that these dynamical changes can be detected by the EPST method. Finally, two artificial neural network was trained using the EPST features to estimate the faulty parameters of the system. It was shown that the EPST features can be used as effective indicators for characterizing the nonlinear response of the system even in the multi-periodic or quasi-periodic domain with complex nonlinearities.

In this study, the proposed method was only applied to numerical data obtained from the mathematical model of the system. Although the results were promising, there is no guarantee that we can obtain the same prediction accuracy for real experimental data. Hence, it is of importance to confirm the effectiveness of the approach with experimental analysis. In addition, only two parametric defects (defects due to change of parameter values) were considered in this paper, whereas in real world applications we might have multiple parametric defects in the system or even defects of the type that can change the structure of the mathematical model of the system. 

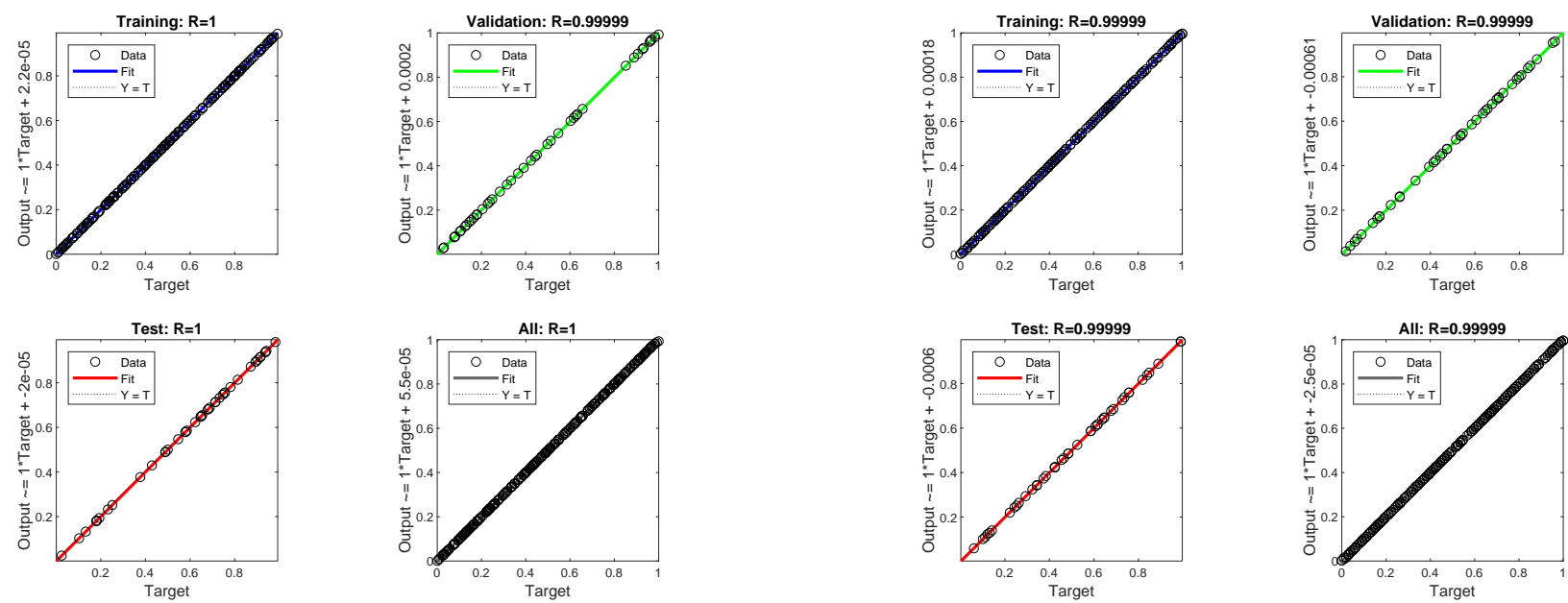

(a)

(b)

Figure 6. Training regression of ANNs in prediction of periodic input for a) $K_{i}$ b) $f_{s}$

Table 1. ANNs fault identification results

\begin{tabular}{|c|c|c|c|c|c|c|c|c|}
\hline \multirow[t]{2}{*}{ No } & & \multirow{2}{*}{$\begin{array}{l}\text { Actual } \\
\text { Value }\end{array}$} & \multicolumn{2}{|c|}{ Periodic } & \multicolumn{2}{|c|}{ Bi-Periodic } & \multicolumn{2}{|c|}{ Quasi-Periodic } \\
\hline & & & Value & Error $\%$ & Value & Error $\%$ & Value & Error $\%$ \\
\hline \multirow{2}{*}{1} & $K_{i}$ & 0.5001 & 0.5000 & 0.02 & 0.5003 & 0.04 & 0.5004 & 0.04 \\
\hline & $f_{s}$ & 81.0000 & 80.9802 & 0.02 & 81.0352 & 0.04 & 80.9280 & 0.09 \\
\hline \multirow{2}{*}{2} & $K_{i}$ & 0.1934 & 0.1934 & 0.01 & 0.1929 & 0.27 & 0.1934 & 0.03 \\
\hline & $f_{s}$ & 27.0000 & 26.5399 & 1.70 & 26.3674 & 2.34 & 27.0846 & 0.31 \\
\hline \multirow{2}{*}{3} & $K_{i}$ & 0.5646 & 0.5642 & 0.08 & 0.5649 & 0.05 & 0.5654 & 0.13 \\
\hline & $f_{s}$ & 49.0000 & 48.9866 & 0.03 & 48.8029 & 0.40 & 49.0284 & 0.06 \\
\hline \multirow{2}{*}{4} & $K_{i}$ & 0.1828 & 0.1828 & 0.04 & 0.1826 & 0.10 & 0.1828 & 0.00 \\
\hline & $f_{s}$ & 70.0000 & 69.9075 & 0.13 & 69.9971 & 0.00 & 69.9037 & 0.14 \\
\hline \multirow{2}{*}{5} & $K_{i}$ & 0.1728 & 0.1729 & 0.08 & 0.1724 & 0.21 & 0.1728 & 0.04 \\
\hline & $f_{s}$ & 25.0000 & 24.4414 & 2.23 & 24.3327 & 2.67 & 25.1959 & 0.78 \\
\hline \multirow{2}{*}{6} & $K_{i}$ & 0.1482 & 0.1481 & 0.07 & 0.1486 & 0.27 & 0.1486 & 0.28 \\
\hline & $f_{s}$ & 35.0000 & 34.8031 & 0.56 & 35.5636 & 1.61 & 35.4071 & 1.16 \\
\hline \multirow{2}{*}{7} & $K_{i}$ & 0.3234 & 0.3234 & 0.00 & 0.3234 & 0.01 & 0.3234 & 0.01 \\
\hline & $f_{s}$ & 67.0000 & 66.9028 & 0.15 & 67.1100 & 0.16 & 67.1874 & 0.28 \\
\hline \multirow{2}{*}{8} & $K_{i}$ & 0.5524 & 0.5523 & 0.03 & 0.5525 & 0.00 & 0.5525 & 0.01 \\
\hline & $f_{s}$ & 63.0000 & 63.1198 & 0.19 & 62.6932 & 0.49 & 62.6613 & 0.54 \\
\hline \multirow{2}{*}{9} & $K_{i}$ & 0.4575 & 0.4575 & 0.01 & 0.4576 & 0.01 & 0.4575 & 0.00 \\
\hline & $f_{s}$ & 44.0000 & 43.8932 & 0.24 & 44.0651 & 0.15 & 44.1911 & 0.43 \\
\hline \multirow{2}{*}{$\begin{array}{c}\text { Mean } \\
\text { Error \%o }\end{array}$} & $K_{i}$ & & \multicolumn{2}{|c|}{0.04} & \multicolumn{2}{|c|}{0.11} & \multicolumn{2}{|c|}{0.06} \\
\hline & $f_{s}$ & & \multicolumn{2}{|c|}{0.58} & \multicolumn{2}{|c|}{0.87} & \multicolumn{2}{|c|}{0.42} \\
\hline
\end{tabular}




\section{ACKNOWLEDGMENT}

This work is supported by the US Office of Naval Research under the grant ONR N0014-15-1-2311 with Capt. Lynn Petersen as the Program Manager. We deeply appreciate this support and are humbled by ONR's enthusiastic recognition of the importance of this research.

\section{NOMENCLATURE}

\section{Electro-Hydraulic Servo System}

\begin{tabular}{|c|c|c|c|}
\hline$a$ & Width of spool edges & $\mathrm{m}$ & $4 e-03$ \\
\hline$A$ & Area of air gap & $\mathrm{m}^{2}$ & \\
\hline$A_{5}$ & Drain orifice area & $\mathrm{m}^{2}$ & \\
\hline$A_{L}$ & $\begin{array}{l}\text { Area of the flow between spool } \\
\text { and sleeve edges } \mathrm{m}^{2}\end{array}$ & & \\
\hline$A_{o}$ & Orifice area & $\mathrm{m}^{2}$ & \\
\hline $\begin{array}{ll}A_{a^{\prime}}, & A_{b^{\prime}}, \\
A_{c^{\prime}}, & \text { and } \\
A_{y^{\prime}} & \end{array}$ & Spool valve restrictions areas & $\mathrm{m}^{2}$ & \\
\hline$A_{P}$ & Piston area & $\mathrm{m}^{2}$ & $7 e-04$ \\
\hline$A_{s}$ & Spool cross-sectional area & $\mathrm{m}^{2}$ & \\
\hline$b$ & Width of sleeve slots & $\mathrm{m}$ & $4 e-03$ \\
\hline$B$ & Bulk modulus of oil & $\mathrm{Pa}$ & $1.5 \mathrm{e} 09$ \\
\hline$c$ & Spool radial clearance & $\mathrm{m}$ & $2 \mathrm{e}-06$ \\
\hline$C_{c}$ & Contraction coefficient & & \\
\hline$C_{d}$ and $C_{D}$ & Discharge coefficients & & 0.661 \\
\hline$d_{f}$ & Flapper nozzle diameter & $\mathrm{m}$ & $5 e-04$ \\
\hline$d_{5}$ & Diameter of return orifice & $\mathrm{m}$ & $6 e-04$ \\
\hline$d_{s}$ & Spool diameter & $\mathrm{m}$ & $4.6 \mathrm{e}-03$ \\
\hline$f_{\theta}$ & Armature damping coefficient & $\mathrm{Nms} / \mathrm{rad}$ & 0.002 \\
\hline$F_{j}$ & Hydraulic momentum force & $\mathrm{N}$ & \\
\hline$f_{p}$ & Piston friction coefficient & $\mathrm{Ns} / \mathrm{m}$ & 1000 \\
\hline$f_{s}$ & Spool friction coefficient & $\mathrm{Ns} / \mathrm{m}$ & 3.05 \\
\hline$F_{s}$ & $\begin{array}{l}\text { Force acting at the extremity of } \\
\text { the feedback spring }\end{array}$ & $\mathrm{N}$ & \\
\hline$H$ & $\begin{array}{l}\text { Magneto-motive force per unit } \\
\text { length }\end{array}$ & $\mathrm{A} / \mathrm{m}$ & \\
\hline$i_{b}$ & Feedback current & $\mathrm{A}$ & \\
\hline$i_{c}$ & Control current & $\mathrm{A}$ & \\
\hline$i_{e}$ & Torque motor input current & $\mathrm{A}$ & \\
\hline$J$ & $\begin{array}{l}\text { Moment of inertia of rotating } \\
\text { part }\end{array}$ & $\mathrm{Nms}^{2}$ & $5 e-07$ \\
\hline$K_{b}$ & Load coefficient & $\mathrm{N} / \mathrm{m}$ & 0 \\
\hline$K_{F B}$ & Feedback gain & $\mathrm{A} / \mathrm{m}$ & 1 \\
\hline$K_{L f}$ & Equivalent flapper seat stiffness & $\mathrm{N} / \mathrm{m}$ & $1 \mathrm{e} 6$ \\
\hline$K_{i}$ & Current-torque gain & $\mathrm{Nm} / \mathrm{A}$ & 0.559 \\
\hline$K_{s}$ & Stiffness of the feedback spring & $\mathrm{N} / \mathrm{m}$ & 900 \\
\hline$K_{T}$ & Stiffness of flexure tube & $\mathrm{Nm} / \mathrm{rad}$ & 10.68 \\
\hline$K$ & Rotational angle-torque gain & $\mathrm{Nm} / \mathrm{rad}$ & $9.45 \mathrm{e}-4$ \\
\hline$L$ & Armature length & $\mathrm{m}$ & 0.029 \\
\hline$L_{f}$ & Flapper length & $\mathrm{m}$ & 0.009 \\
\hline$L_{s}$ & Length of the feedback spring & $\mathrm{m}$ & 0.03 \\
\hline
\end{tabular}

Length of the feedback spring $\mathrm{m}$
Length of spool land

Piston mass

Spool mass

Pressure in the left side of the

flapper valve

$P_{2} \quad$ Pressure in the right side of the $\mathrm{Pa}$

flapper valve

$P_{3} \quad$ Pressure in the flapper valve re- $\quad \mathrm{Pa}$ turn chamber

$P_{A}$ and $P_{B}$

$P_{s}$

$P_{T}$

$Q$

$Q_{1}$

$Q_{2}$

$Q_{3}$

$Q_{4}$

$Q_{5}$

$Q_{a}, \quad Q_{b}$,

$Q_{c}$, and $Q_{d}$

$R_{i}$

$R_{s}$

$T$

$T_{F}$

$T_{L}$

$T_{P}$

$V_{3}$

$V_{c}$

$V_{o}$

$x$

$x_{a}$

$x_{f}$

$x_{i}$

$x_{o}$

$\lambda$

$\lambda_{p}$

$\mu$

$\mu_{o}$

$\mu_{r}$

$\omega$
Hydraulic cylinder pressures $\quad \mathrm{Pa}$

Supply pressure $\mathrm{Pa}$

Return line pressure

Flow rate

Flow rate in the left orifice

Flow rate in the right orifice

Left flapper nozzle flow rate

Right flapper nozzle flow rate

Flapper valve drain flow rate

Flow rates through the spool

valve restrictions

Resistance to internal leakage $\quad \mathrm{Ns} / \mathrm{m}^{5} \quad 1 \mathrm{e} 20$

Flapper seat damping coeffi- Nms/rad 5000 cient

Torque of electromagnetic Nm torque motor

Feedback torque

Torque due to flapper displace- $\mathrm{Nm}$

ment limiter

Torque due to the pressure $\mathrm{Nm}$

forces

Volume of the flapper valve re- $\mathrm{m}^{3} \quad 5 \mathrm{e}-06$

turn chamber

Half of the volume of oil filling $\mathrm{m}^{3} \quad 1 \mathrm{e}-04$

the cylinder

Initial volume of oil in the spool $\mathrm{m}^{3} \quad 2 \mathrm{e}-06$

side chamber

Spool displacement

$\mathrm{m}$

Displacement of the armature $\mathrm{m}$

end

Flapper displacement on the $\mathrm{m}$

level of the jet nozzles

Flapper displacement limit m

Length of the air gap in the neu- $m \quad 3 e-04$

tral position of armature

Magneto-motive force A

Magneto-motive force of the A

66.75

permanent magnet

Permeability

Permeability of the air

$\mathrm{Vs} / \mathrm{Am}$

Vs/Am 4e-07

Relative permeability

Oil density

$\mathrm{kg} / \mathrm{m}^{3}$

867

Width of ports on the valve $\mathrm{m}$ sleeve

$\mathrm{m}^{3} / \mathrm{s}$

$\mathrm{m}^{3} / \mathrm{s}$

$3 / \mathrm{s}$

$\mathrm{m}^{3} / \mathrm{s}$
$1.5 \mathrm{e}-02$

0.2 


\begin{tabular}{|c|c|}
\hline$\theta$ & Armature rotation angle \\
\hline & EPST Method \\
\hline$\Gamma()$. & Kernel function \\
\hline$\Phi_{m}$ & $\begin{array}{l}\text { Legendre polynomial of order } \\
m\end{array}$ \\
\hline$\Psi$ & $\begin{array}{l}\text { Unknown density distribution } \\
\text { for a given data }\end{array}$ \\
\hline$y_{d}, \hat{\Psi}$ & $\begin{array}{l}\text { Estimated kernel density distri- } \\
\text { bution for a given data }\end{array}$ \\
\hline$\Psi_{a}$ & $\begin{array}{l}\text { Approximated density distribu- } \\
\text { tion for a given data using Leg- } \\
\text { endre polynomials }\end{array}$ \\
\hline$h$ & Bandwidth \\
\hline$\beta$ & $\begin{array}{l}\text { Legendre polynomial coeffi- } \\
\text { cients }\end{array}$ \\
\hline$Z$ & Residual vector \\
\hline
\end{tabular}

\section{REFERENCES}

Baskiotis, C., Raymond, J., \& Rault, A. (1979). Parameter identification and discriminant analysis for jet engine machanical state diagnosis. In 18th IEEE Conference on Decision and Control including the Symposium on Adaptive Processes (Vol. 18, pp. 648-650).

Frank, P., Ding, S. X., \& Koppen-Seliger, B. (2000). Current developments in the theory of FDI. In Proceedings of SAFEPROCESS (Vol. 1, pp. 16-27).

Gordić, D., Babić, M., \& Jovičić, N. (2004). Modelling of spool position feedback servovalves. International Journal of Fluid Power, 5(1), 37-51.

Isermann, R. (1982). Parameter adaptive control algorithmsa tutorial. Automatica, 18(5), 513-528.

Isermann, R. (1984). Process fault detection based on modeling and estimation methodsa survey. automatica, 20(4), 387-404.

Isermann, R. (2005). Model-based fault-detection and diagnosis-status and applications. Annual Reviews in Control, 29(1), 71-85.

Kappaganthu, K., \& Nataraj, C. (2011). Feature selection for fault detection in rolling element bearings using mutual information. Journal of vibration and acoustics, 133(6), 061001.

Liu, X.-Q., Zhang, H.-Y., Liu, J., \& Yang, J. (2000). Fault detection and diagnosis of permanent-magnet DC motor based on parameter estimation and neural network.
IEEE Transactions on Industrial Electronics, 47(5), 1021-1030.

Mohamad, T. H., Kwuimy, C. K., \& Nataraj, C. (2017). Discrimination of multiple faults in bearings using density-based orthogonal functions of the time response. In Asme 2017 international design engineering technical conferences and computers and information in engineering conference (pp. V008T12A035V008T12A035).

Mohamad, T. H., \& Nataraj, C. (2017, October). Gear fault diagnostics using extended phase space topology. In Annual conference of the prognostics and health management society.

Mohamad, T. H., Samadani, M., \& Nataraj, C. (n.d.). Rolling element bearing diagnostics using extended phase space topology. Journal of Vibration and Acoustics.

Rabie, M. (2009). Fluid power engineering. McGraw Hill Professional.

Samadani, M., Behbahani, S., \& Nataraj, C. (2013). A reliability-based manufacturing process planning method for the components of a complex mechatronic system. Applied Mathematical Modelling, 37(24), 9829-9845.

Samadani, M., Kwuimy, C. A., \& Nataraj, C. (2014). Fault detection and severity analysis of servo valves using recurrence quantification analysis. In Annual conference of the prognostics and health management society.

Samadani, M., Kwuimy, C. K., \& Nataraj, C. (2013). Diagnostics of a nonlinear pendulum using computational intelligence. In Asme 2013 dynamic systems and control conference.

Samadani, M., Kwuimy, C. K., \& Nataraj, C. (2015). Modelbased fault diagnostics of nonlinear systems using the features of the phase space response. Communications in Nonlinear Science and Numerical Simulation, 20(2), 583-593.

Samadani, M., Mohamad, T. H., \& Nataraj, C. (2016). Feature extraction for bearing diagnostics based on the characterization of orbit plots with orthogonal functions. In Asme 2016 international design engineering technical conferences and computers and information in engineering conference (pp. V008T10A026V008T10A026). 